\title{
Impactos técnicos y económicos para comercializadores de electricidad debido a la implementación de vehículos eléctricos en Colombia*
}

\author{
Kevin Eduardo Rojas Quiroga** \\ John Fredy Nieto Vargas ${ }^{* * *}$ \\ Geovanny Marulanda****
}

Recibido: 31/05/2017 - Aceptado: 26/09/2018

https://doi.org/10.22395/rium.v18n34a13

\begin{abstract}
Resumen
La necesidad de diversificar la matriz energética colombiana ha generado incentivos que apuntan hacia una mayor participación de los vehículos eléctricos (VE) en el mercado. A medida que aumente la penetración de VE en las ciudades, mayores serán los impactos sobre las redes de distribución de energía eléctrica. En este trabajo, se describe el impacto técnico y económico que podría enfrentar un operador de red o un comercializador de energía en Colombia ante la inclusión de VE en un sistema de distribución. Para esto se simularon tres escenarios de inclusión de VE sobre un sistema de distribución secundario que hace parte de la red Fundadores de Manizales. Los resultados muestran que los impactos técnicos y económicos sobre los operadores de red dependen de la tecnología de los VE, adicional a esto, muestran que los comercializadores podrían resultar beneficiados de su masificación en Colombia.
\end{abstract}

Palabras clave: comercializador de energía eléctrica; operador de red; sistema de distribución; vehículo eléctrico.

Investigación derivada del trabajo de grado titulado Análisis de flujo de potencia y perfiles de tensión en sistemas de distribución ante distintos niveles de inclusión de vehículos eléctricos presentado en la Universidad de La Salle, Bogotá, Colombia.

** Ingeniero electricista. Universidad de La Salle, Colombia. Correo electrónico: krojas16@unisalle.edu.co

*** Ingeniero electricista. Universidad de La Salle, Colombia. Orcid: http://orcid.org/0000-0002-3549-1394 Correo electrónico: jnieto11@unisalle.edu.co.

**** MSc en Ingeniería Eléctrica. Universidad de La Salle, Colombia. Correo electrónico: gemarulanda@unisalle. edu.co. Orcid: http://orcid.org/0000-0002-7799-9890 


\title{
Technical and economical impacts for marketers due to the implementation of electric vehicles in Colombia
}

\begin{abstract}
The need for diversification in the Colombian energetical matrix have produced incentives towards greater participation of electric vehicles (EV) in the market. As EV in cities get deeper into the market the greater the impact over the electrical distribution networks will be. In this paper are described the technical and economic impacts that a network operator or energy provider might face in their distribution systems because of the entrance of EV to the Colombian market. For this, three scenarios were simulated over a secondary distribution system belonging to the Fundadores de Manizales network. The results show that the economic and technical impacts among the network operators depend on the Ev technologies, and additionally, show that the marketers might be benefited from its massification in Colombia.
\end{abstract}

Keywords: electrical energy marketer; network operator; distribution system; electric vehicle.

\section{Impactos técnicos e econômicos para comercializadores de eletricidade devido à implantação de veículos elétricos na Colômbia}

\begin{abstract}
Resumo
A necessidade de diversificar a matriz energética colombiana tem gerado incentivos que apontam a maior participação dos veículos elétricos (VE) no mercado. À medida que aumentar a inserção desses veículos nas cidades, maiores serão os impactos sobre as redes de distribuição de energia elétrica. Neste trabalho, descreve-se o impacto técnico e econômico que poderia enfrentar um operador de rede ou um comercializador de energia na Colômbia diante da inclusão de VE em um sistema de distribuição. Para isso, foram simulados três cenários de inclusão de VE sobre um sistema de distribuição secundário que faz parte da rede Fundadores de Manizales, Colômbia. Os resultados mostram que os impactos técnicos e econômicos sobre os operadores de rede dependem da tecnologia dos VE; além disso, indicam que os comercializadores poderiam ser beneficiados de sua massificação na Colômbia.
\end{abstract}

Palavras-chave: comercializador de energia elétrica; operador de rede; sistema de distribuição; veículo elétrico. 


\section{INTRODUCCIÓN}

Los beneficios de cambiar los vehículos de combustión interna por vehículos eléctricos (VE) son diversos. Desde un punto de vista ambiental, se obtienen reducciones en las emisiones de dióxido de carbono y óxido de nitrógeno, se diversifica la matriz energética de un país y se mitigan los impactos del cambio climático. Además de esto, podrían alcanzarse reducciones de hasta el $80 \%$ en los costos de operación cuando se emplean VE en lugar de vehículos de combustión interna [1-2].

Estos beneficios han orientado políticas que buscan aumentar la accesibilidad a los VE. Entre los mecanismos que se han implementado se encuentran las disminuciones en los impuestos de compra y circulación, y permisos de acceso a zonas restringidas para vehículos de transporte particular. Con estas medidas, al cierre del año 2015, cerca de 1,26 millones de VE se encontraban en circulación, China y Estados Unidos son líderes en su uso con más del 50 \% del mercado mundial [1]. De manera análoga, distintas iniciativas indican que la participación de VE en el mercado del transporte seguirá creciendo. Por ejemplo, EVI (Electric Vehicles Initiative por sus siglas en inglés), plantea la necesidad de contar con 20 millones de VE para el 2020, mientras que la Declaración de París de electro-movilidad y cambio climático y llamado a la acción, establece como objetivo 100 millones de VE para el año 2030 [1, 3].

Colombia no ha sido ajena a la necesidad de fortalecer el mercado de los VE. Desde el año 2011, el gobierno nacional aprobó la importación de 50 taxis eléctricos por medio de un convenio liderado por la empresa Codensa-Emgesa con el propósito de realizar pruebas de desempeño de los vehículos eléctricos [4]. En diciembre del año 2013 se aprobó el Decreto 2909 con una vigencia de tres años, y se autorizó el ingreso de $750 \mathrm{VE}$ híbridos de tipo enchufable cada año al país [5]. En ese mismo año en Bogotá se aprobó el Decreto 575 en el que se eliminan para los VE las restricciones de circulación en el área urbana para automotores en los horarios con mayor afluencia de tráfico [4]. De manera análoga, en [6] se identifica la necesidad de diversificar la canasta energética colombiana a través de la masificación de VE en el país, llamando la atención sobre la necesidad de transformar el mercado eléctrico y generar cambios institucionales que permitan la inclusión de VE además de proponer como estrategias de inclusión, la promoción de VE para transporte público, y la implementación de planes que promuevan el uso de energéticos alternativos para los vehículos.

Conforme aumenta la penetración de VE en las ciudades, mayores son los impactos sobre las redes de distribución. En [7] se muestra que las pérdidas en un sistema de distribución podrían incrementarse hasta en un $40 \%$ en las horas fuera de pico y aumentar los costos de expansión del sistema en un $15 \%$ debido a la carga de VE en horas pico. Por su parte, los resultados presentados en [8] muestran que el impacto de 
los VE en las tensiones y las pérdidas en un sistema de distribución es menor cuando se realiza la carga de los VE de forma coordinada. Por otro lado, en [9] se determina el impacto de los VE en los límites de tensión, la calidad de la potencia y el desbalance en sistemas de distribución. Finalmente, en [10] se propone un modelo para determinar el impacto de los tiempos de carga y descarga de los VE en las pérdidas y en la cargabilidad del sistema cuando se tienen en cuenta las preferencias en los tiempos de carga de los usuarios.

Entre las alternativas de masificación de VE que se proponen para América Latina se encuentran los incentivos regulatorios y las tarifas variables aplicables a VE que promuevan su recarga en periodos distintos a las horas pico [11]. Sin embargo, los operadores de red (OR) y comercializadores podrían presentar actualmente beneficios económicos y técnicos que los motiven a impulsar la masificación de VE. Entre los beneficios que podrían obtenerse se encuentran [10-11]: i) aumento de los ingresos vía tarifa por incremento de la demanda de energía, ii) disminuciones en los costos de compra de energía a precio de bolsa, iii) mejoras en los perfiles de tensión en determinadas horas del día, iv) disminución de la corriente en conductores, y v) aumento en la confiabilidad del sistema.

En el caso de Colombia, los OR se encargan de la operación de los sistemas de distribución, los comercializadores de las actividades de compra y venta de energía, y ambos pueden o no estar integrados en la misma empresa [12-13]. De esta forma, se espera que los aspectos técnicos de la inclusión de VE afecten en mayor medida las actividades de los OR y los aspectos económicos las actividades de los comercializadores. Con el ánimo de brindar las dos perspectivas, en este trabajo se asume que ambos hacen parte de la misma empresa presentando los impactos técnicos y económicos que enfrentaría un comercializador -OR ante la implementación de VE en un sistema de distribución. Esta empresa integrada se denominará en adelante como comercializador.

Para presentar los resultados obtenidos, este trabajo se encuentra ordenado como sigue: La sección 1 presenta la metodología propuesta, la sección 2 los resultados obtenidos al simular la metodología sobre un sistema de prueba monofásico de 26 nodos, y en la sección 3 se realiza una discusión de los resultados obtenidos.

\section{METODOLOGÍA}

Para determinar el impacto que tendría la inclusión de VE sobre la operación de un sistema de distribución, se evaluaron tres modelos distintos que procuran la maximización del beneficio de un comercializador. En el primer modelo, se consideran las restricciones técnicas del sistema sin considerar la participación de VE en la red. En el segundo, se incluye el consumo de energía eléctrica debido a la carga de los VE. 
Por último, en el tercer modelo, se adiciona la posibilidad de que los VE entreguen energía almacenada al sistema.

Modelo 1: maximización del beneficio del comercializador sin inclusión de VE.

Las ecuaciones de la (1) a la (8) presentan el modelo de optimización que maximiza el beneficio de un comercializador, considerando la operación en estado estable de un sistema de distribución [14-15]:

$$
\begin{gathered}
\max \left[\sum_{t=1}^{T} \sum_{n=1}^{N}\left[\delta_{n} P d_{n, t}\right]-\sum_{t=1}^{T} \sum_{n=1}^{N}\left[\rho_{t} P d_{n, t}\right]-\sum_{t=1}^{T} \sum_{l=1}^{L}\left[\rho_{t} R_{l} I_{l, t}^{2}\right]\right] \\
\sum_{l=1}^{L} P_{l, t}^{\text {in }}+P g_{n, t}=\sum_{l=1}^{L}\left[P_{l, t}^{\text {out }}+R_{l} I_{l, t}\right]+P d_{n, t} \quad \forall n=1,2, \ldots, N ; \forall t=1,2, \ldots, T \\
\sum_{l=1}^{L} Q_{l, t}^{\text {in }}+Q g_{n, t}=\sum_{l=1}^{L}\left[Q_{l, t}^{\text {out }}+X_{l} I_{l, t}^{2}\right]+Q d_{n, t} \quad \forall n=1,2, \ldots, N ; \forall t=1,2, \ldots, T \\
V_{l . t}^{\text {in } 2}-V_{l . t}^{\text {out } 2}=2\left[R_{l} P_{l, t}^{\text {out }}+X_{l} Q_{l, t}^{\text {out }}\right]+Z_{l}^{2} I_{l, t}^{2} \quad \forall l=1,2, \ldots, L ; \forall t=1,2, \ldots, T \\
V_{l . t}^{\text {out } 2} I_{l, t}^{2}=P_{l, t}^{\text {out } 2}+Q_{l, t}^{\text {out } 2} \quad \forall l=1,2, \ldots, L ; \forall t=1,2, \ldots, T \\
\underline{V}_{n} \leq V_{n, t} \leq \bar{V}_{n} \forall n=1,2, \ldots, N ; \forall t=1,2, \ldots, T \quad 0 \leq I_{l, t} \leq \bar{I}_{l} \\
\forall l=1,2, \ldots, N ; \forall t=1,2, \ldots, T \\
0 \leq P g \leq \overline{P g}
\end{gathered}
$$

Donde:

l,L I Índice y número total de líneas.

$n, N \quad$ Índice y número total de nodos.

$t, T \quad$ Índice y número total de horas.

$\delta_{n} \quad$ Tarifa del nodon.

$\rho_{t} \quad$ Precio de bolsa en la hora $t$.

$R_{l}, X_{l}, Z_{l} \quad$ Resistencia, reactancia y magnitud de la impedancia de la línea $l$.

$P g_{n, t}, P d_{n, t}$ Potencia activa generada y demandada en el nodo $n$ en la hora $t$.

$P_{l, t}^{\text {in }}, P_{l, t}^{\text {out }} \quad$ Potencia activa de entrada en los nodos de envío y recibo de la línea $l$ en la hora $t$. 
$Q g_{n, t}, Q d_{n, t}$ Potencia reactiva generada y demandada en el nodo $n$ en la hora $t$.

$Q_{l, t}^{\text {in }}, Q_{l, t}^{\text {out }} \quad$ Potencia reactiva de entrada en los nodos de envío y recibo de la línea $l$ en la hora $t$.

$I_{l, t} \bar{I}_{l} \quad$ Corriente y corriente máxima de la línea $l$ en la hora $t$.

$V_{l . t}^{\text {in }}, V_{l . t}^{\text {in }} \quad$ Tensión en los nodos de envío y recibo de la línea $l$ en la hora $t$.

$V_{n, t} \quad$ Tensión en el nodo $n$ durante en la hora $t$.

$\underline{V}_{n}, \bar{V}_{n} \quad$ Tensiones mínima y máxima en el nodo $n$.

$P g, \overline{P g} \quad$ Potencia y potencia máxima en el transformador de distribución.

La ecuación (1) representa la función objetivo y se divide en tres componentes. El primero, corresponde a los ingresos que recibe el comercializador a través de la tarifa. Para el caso colombiano, esta tarifa depende del estrato socioeconómico y del producto entre el consumo mensual de energía eléctrica y el costo unitario de prestación del servicio [16]. Los componentes segundo y tercero, se encuentran relacionados con la compra de la energía necesaria para suplir la demanda y las pérdidas del sistema a precio de bolsa. Este precio varía a lo largo del día con una resolución horaria.

Cabe aclarar, que el costo de las pérdidas es remunerado de forma parcial en la fórmula empleada en el cálculo del costo unitario de prestación del servicio [16]. Esta fórmula se aplica a los OR y depende de la demanda y de las pérdidas del sistema, por lo que el término $\delta_{n}$ podría variar de un mes a otro. En este trabajo se asume el costo unitario de prestación del servicio como un valor constante para cada nodo dado que la operación del sistema se simula para un único día. Nótese además que las pérdidas del sistema se han incluido como parte de las compras de energía que debe realizar el comercializador a precio de bolsa.

Por otro lado, las restricciones de las ecuaciones (2) y (3) corresponden al balance de potencia activa y reactiva en cada nodo. Las restricciones de las ecuaciones (4) y (5) relacionan las magnitudes de las tensiones en cada nodo, con las magnitudes de las corrientes y el flujo de potencia activa y reactiva por cada circuito. Las restricciones de las ecuaciones (6) y (7) corresponden a los límites de tensión en cada nodo y los límites de corriente en las líneas. Finalmente, en la ecuación (8), se ha tenido en cuenta la potencia máxima en el alimentador del sistema de distribución.

\section{Modelo 2: maximización del beneficio del comercializador considerando carga de VE.}

Para incluir el impacto de la carga de los VE en el sistema de distribución se han modificado las ecuaciones (1) y (2) del modelo 1 y se han adicionado tres restricciones. 
Las ecuaciones de la (9) a la (13), presentan las modificaciones y las restricciones adicionadas:

$$
\begin{gathered}
\max \left[\sum_{t=1}^{T} \sum_{n=1}^{N}\left[\delta_{n}\left(P d_{n, t}+P v_{n, t}\right)\right]-\sum_{t=1}^{T} \sum_{n=1}^{N}\left[\rho_{t}\left(P d_{n, t}+P v_{n, t}\right)\right]-\sum_{t=1}^{T} \sum_{l=1}^{L}\left[\rho_{t} R_{l} I_{l, t}^{2}\right]\right] \\
\sum_{l=1}^{L} P_{l, t}^{i n}+P g_{n, t}=\sum_{l=1}^{L}\left[P_{l, t}^{\text {out }}+R_{l} I_{l, t}^{2}\right]+P d_{n, t}+P v_{n, t} \\
\forall n=1,2, \ldots, N ; \forall t=1,2, \ldots, T \\
P v_{n, t}=\sum_{u=1}^{U} \eta_{n, u} P v_{n, t, u} \forall n=1,2, \ldots, N ; \forall t=1,2, \ldots, T \\
0 \leq P v_{n, t, u} \leq{\overline{P v_{n, t, u}}}^{T} \forall n=1,2, \ldots, N ; \forall t=1,2, \ldots, T ; \forall u=1,2, \ldots, U \\
\sum_{t=1}^{T} P v_{n, t, u}=\overline{E v}_{n, u} \forall n=1,2, \ldots, N ; \forall u=1,2, \ldots, U
\end{gathered}
$$

Donde:

u,U I Índice y número total de usuarios.

$\eta_{n, u} \quad$ Rendimiento de la batería del usuario $u$ conectado en el nodo $n$.

$\overline{E v}_{n, u} \quad$ Máxima energía de almacenamiento de la batería del usuario $u$ conectado al nodo $n$.

$P v_{n, t} \quad$ Potencia activa demandada por los VE del nodo $n$ en la hora $t$.

$P v_{n, t, u} \quad$ Potencia activa demandada por el VE del usuario $u$, del nodo $n$ en la hora $t$.

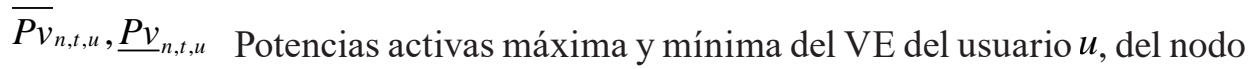
$n$ en la hora $t$.

En la ecuación (9) se ha incluido la potencia demandada por los VE en los ingresos por tarifa y en la compra de energía a precio de bolsa. En la ecuación (10) se incluye la demanda de los VE en el balance de potencia nodal. En la ecuación (11) se establece la potencia demandada por los VE en un nodo como la suma de las potencias demandadas por todos los usuarios que cuentan con VE en ese nodo multiplicado por el rendimiento de cada batería. En la ecuación (12) se tiene en cuenta el límite máximo de carga por hora de la batería de cada VE. Finalmente, en la ecuación (13), se tiene en cuenta el límite máximo de almacenamiento de energía de las baterías de los VE. 
Modelo 3: maximización del beneficio del comercializador considerando carga y descarga en VE.

Empleando la tecnología V2G (siglas derivadas de las palabras vehicle-to-grid del inglés) los cargadores de VE pueden operar de forma bidireccional, esto es, descargar la energía almacenada en las baterías de los VE sobre la red [17]. Para incluir el impacto de la descarga de los VE en el sistema de distribución se han modificado las ecuaciones (12) y (13) del modelo 2, según las ecuaciones (14) y (15):

$$
\begin{gathered}
-{\underline{P v_{n, t, u}}} \leq P v_{n, t, u} \leq \overline{P V}_{n, t, u} \\
\forall n=1,2, \ldots, N ; \forall t=1,2, \ldots, T ; \forall u=1,2, \ldots, U \\
0 \leq \sum_{t=1}^{T} P v_{n, t, u} \leq \overline{E v}_{n, u} \quad \forall n=1,2, \ldots, N ; \forall u=1,2, \ldots, U
\end{gathered}
$$

La ecuación (14) permite a la variable asociada a la potencia demandada por los VE tomar valores negativos y comportarse como inyecciones de potencia a la red. De igual forma, con (15) se garantiza que la energía entregada al sistema por cada VE no sea mayor que la energía almacenada por el mismo a lo largo del día.

Note que los modelos propuestos parten de dos consideraciones, la primera es que los VE deben cargarse completamente al final del periodo de estudio, lo cual se evidencia a través de la ecuación (13). Lo anterior con la intención de analizar el caso de mayor demanda diaria que podría presentarse debido a la inclusión de los VE. La segunda, es que la respuesta que entrega el modelo presenta un escenario positivo para el comercializador dado que le permite encontrar los tiempos de carga y descarga para los VE que le generarían el mayor beneficio. Esta segunda consideración, implica que no se han tenido en cuenta los hábitos de carga y descarga de los VE que podrían presentar los usuarios, ni la necesidad de tener un porcentaje de carga en una hora determinada. El estudio de los hábitos de carga y su inclusión en los modelos propuestos se escapa del alcance de este trabajo, y se propone para ser desarrollado en trabajos futuros.

\section{RESULTADOS}

Para determinar el impacto técnico y económico que enfrentaría un comercializador debido a la inclusión de VE, se plantean tres casos de estudio. En cada caso se analiza el perfil de tensión en los nodos de uno de los ramales del circuito, las pérdidas del sistema y los beneficios económicos obtenidos por el comercializador para un periodo de 24 horas. En el primer caso, se analiza un sistema de distribución que opera sin VE, en el segundo, se incluye consumo de energía debido a las baterías de los VE y, en el tercer caso, se analiza el impacto de la carga y descarga de los VE, esto es, se asume que los VE cuentan con tecnología V2G. Los resultados se han obtenido empleando el software GAMS [18]. 
Como sistema de prueba, se simula el comportamiento de la demanda residencial y de los VE a lo largo de un día, sobre un sistema de distribución secundario que hace parte de la red Fundadores de Manizales [19]. La topología de la red se puede observar en la figura 1. El sistema consta de 26 nodos, cada nodo cuenta con una cantidad de usuarios representada por el número descrito dentro del círculo. De igual forma, la nomenclatura empleada para cada nodo se muestra en la figura. El sistema es radial y opera a tensiones de $240 \mathrm{~V}$ entre fases, y de $120 \mathrm{~V}$ entre fase y neutro. La potencia nominal del transformador es de $50 \mathrm{kVA}$ y alimenta un total de 77 usuarios de clase media. Por otro lado, la figura 2 muestra las curvas de carga del sistema obtenidas para cada caso de estudio. La línea gris representa la demanda total del circuito sin VE, y los puntos por encima y por debajo de esta curva, indican las horas de carga y descarga de los VE obtenidos en cada caso.

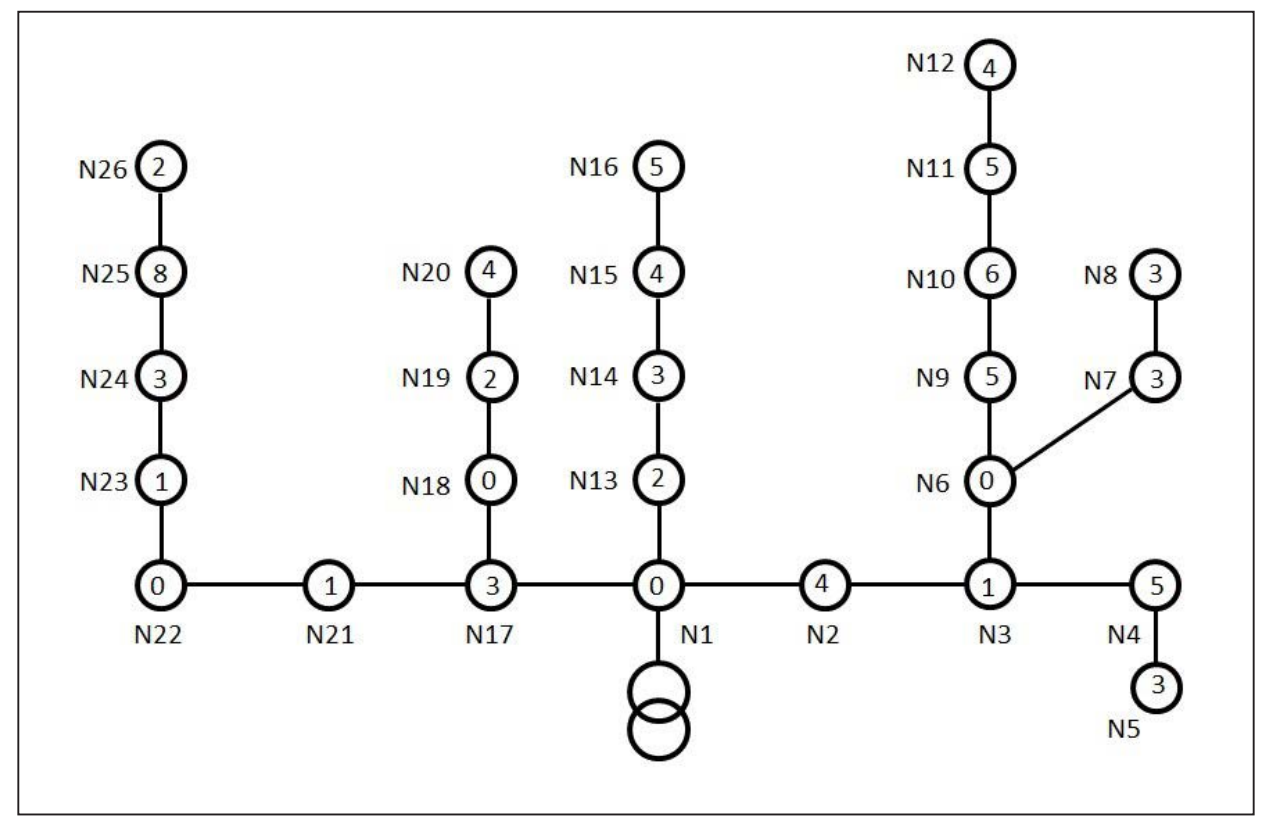

Figura 1. Diagrama unifilar del sistema de prueba

Fuente: elaboración propia.

En este trabajo se ha asumido una tarifa de $346 \mathrm{COP} / \mathrm{kWh}$ para cada usuario, este es el costo unitario de prestación del servicio reportado por un reconocido OR para usuarios estrato 4. Por otro lado, el precio en bolsa ha sido tomado de la base de datos de XM (el operador del Sistema Interconectado Nacional en Colombia) para un día típico del mes en que se tomó el valor de la tarifa [20]. Finalmente, el sistema de prueba empleado en este trabajo presenta diferencias con respecto al presentado en [19]. Los datos completos del sistema pueden ser consultados en [21]. 


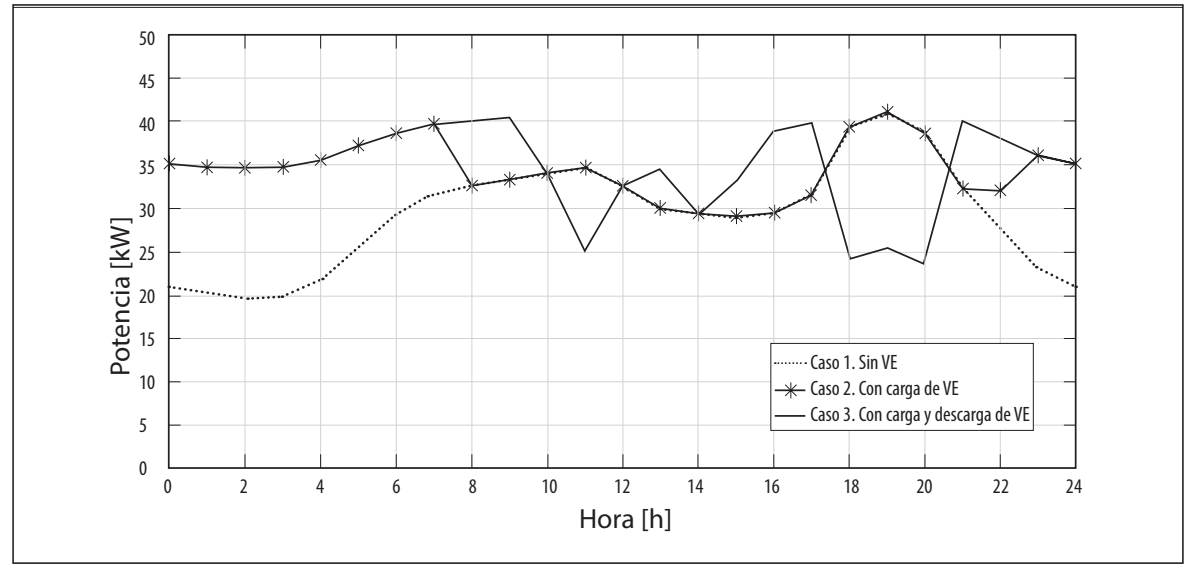

Figura 2. Curva de carga para cada caso de estudio Fuente: elaboración propia.

\section{Caso 1: operación sin VE.}

En este caso se analizó el sistema de distribución sin VE, esto es, se empleó el modelo 1 de la sección 1. Las figuras de la 3 a la 5 presentan los resultados para los perfiles de tensión, las pérdidas totales del sistema y los beneficios del comercializador de forma horaria. La figura 3 presenta los valores en por unidad de las tensiones del ramal que se forma al unir los nodos N1 a N12, resultando este de interés por presentar el mayor número de usuarios. En dicha figura puede notarse que la tensión en los nodos disminuye en los periodos de alta demanda, esto es, cerca del mediodía y entre las horas 18 y 20, lo que presenta un aumento en los periodos de baja demanda.

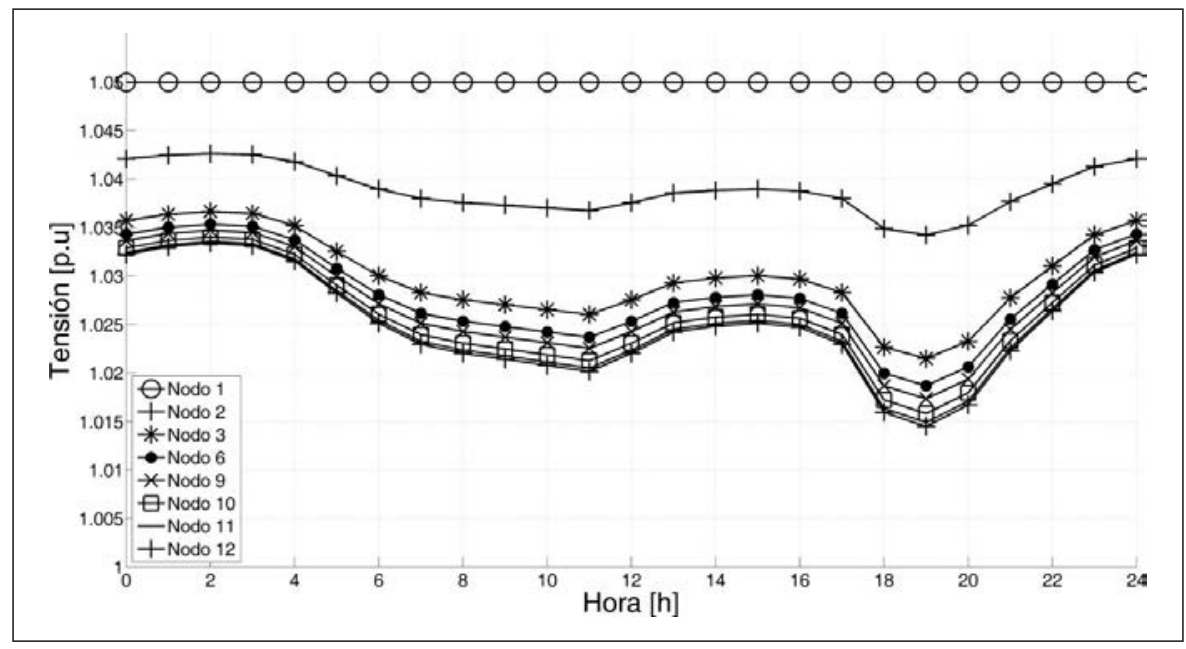

Figura 3. Perfiles de tensión sin VE Fuente: elaboración propia. 


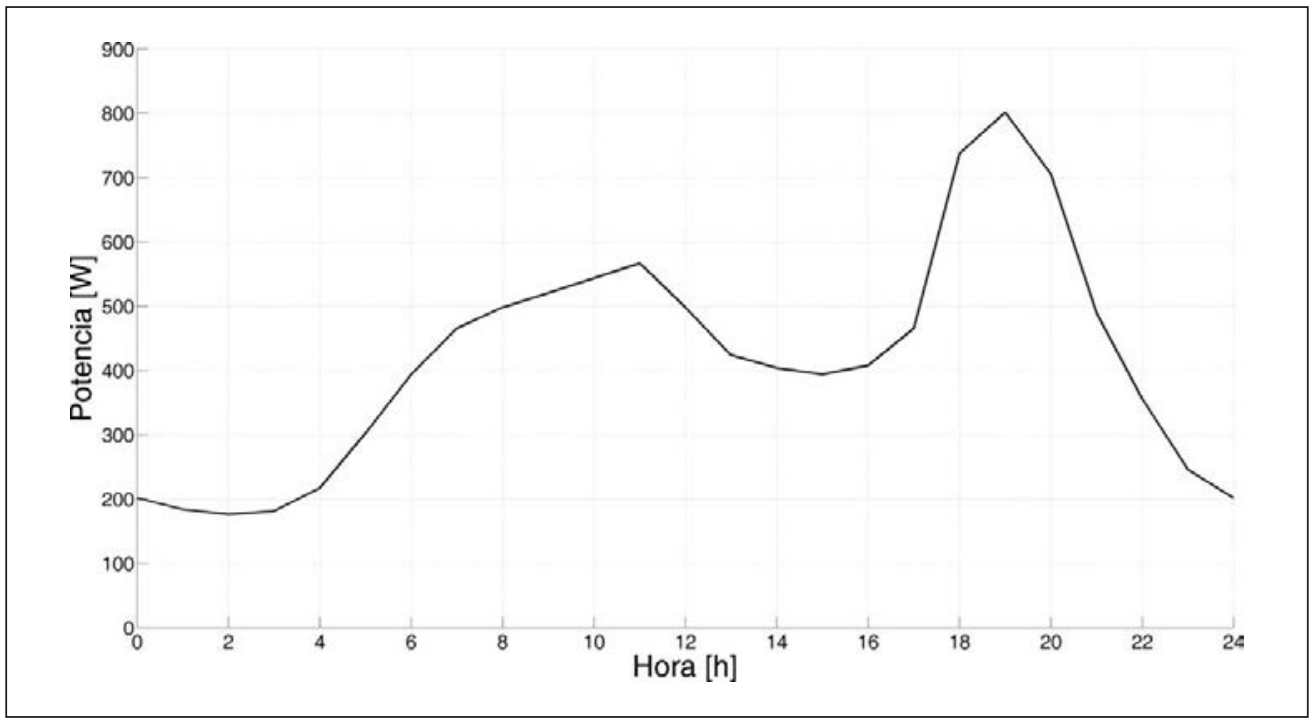

Figura 4. Pérdidas totales sin VE

Fuente: elaboración propia.

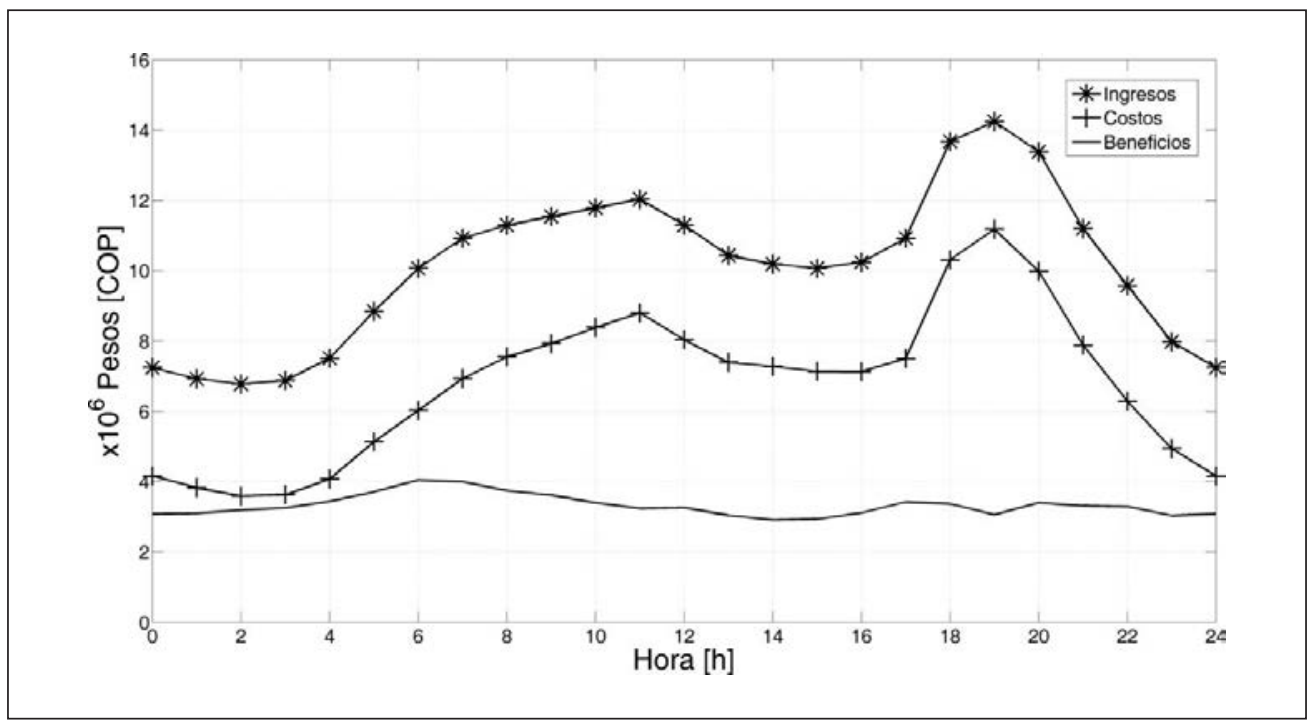

Figura 5. Beneficios del comercializador sin VE

Fuente: elaboración propia.

De manera análoga, la figura 4 muestra las pérdidas de todo el sistema a lo largo del día. Note que las horas de mayores pérdidas coinciden con las de mayor caída de tensión. En este caso las pérdidas diarias de energía ascienden a 10 kWh. Por su parte, la figura 5 presenta el comportamiento de los beneficios del comercializador, 
y su relación con los ingresos por tarifa y los costos de la compra de energía en bolsa. Nótese de esta figura que, aunque la tarifa es un valor fijo y el precio en bolsa es variable, ambas curvas presentan características y beneficios similares para cada hora del día. Los ingresos diarios corresponden a 245 millones de pesos colombianos (MCOP), los costos de la compra de la energía consumida por la demanda 165 MCOP y el comercializador obtiene 80 MCOP diarios en beneficios.

\section{Caso 2: operación incluyendo carga de VE.}

En este caso se analizó el sistema de distribución cuando se asume una inclusión de VE del $20 \%$ en el sistema de distribución y se empleó el modelo 2 presentado en la sección 1. Para esto, se asumió la presencia de un VE en cada nodo del ramal N1-N12 excepto en los nodos N3 y N6 por presentar un bajo número de usuarios. Adicional a esto, se asumieron vehículos de carga lenta con una potencia de $4,8 \mathrm{~kW}$, un rendimiento de las baterías del $100 \%$ y un tiempo de carga de 6 horas. Los perfiles de tensión para las 24 horas del día se presentan en la figura 6. A diferencia de los resultados presentados en la figura 3 para el caso 1, se evidencia una caída de tensión en todos los nodos del ramal entre las horas 0-7 y 21-24. Estos resultados concuerdan con el incremento en las pérdidas que se registran en la figura $7 \mathrm{e}$ indican un incremento en el consumo debido a la conexión de VE en esos periodos. En este caso las pérdidas diarias de energía ascienden a los 15,5 kWh.

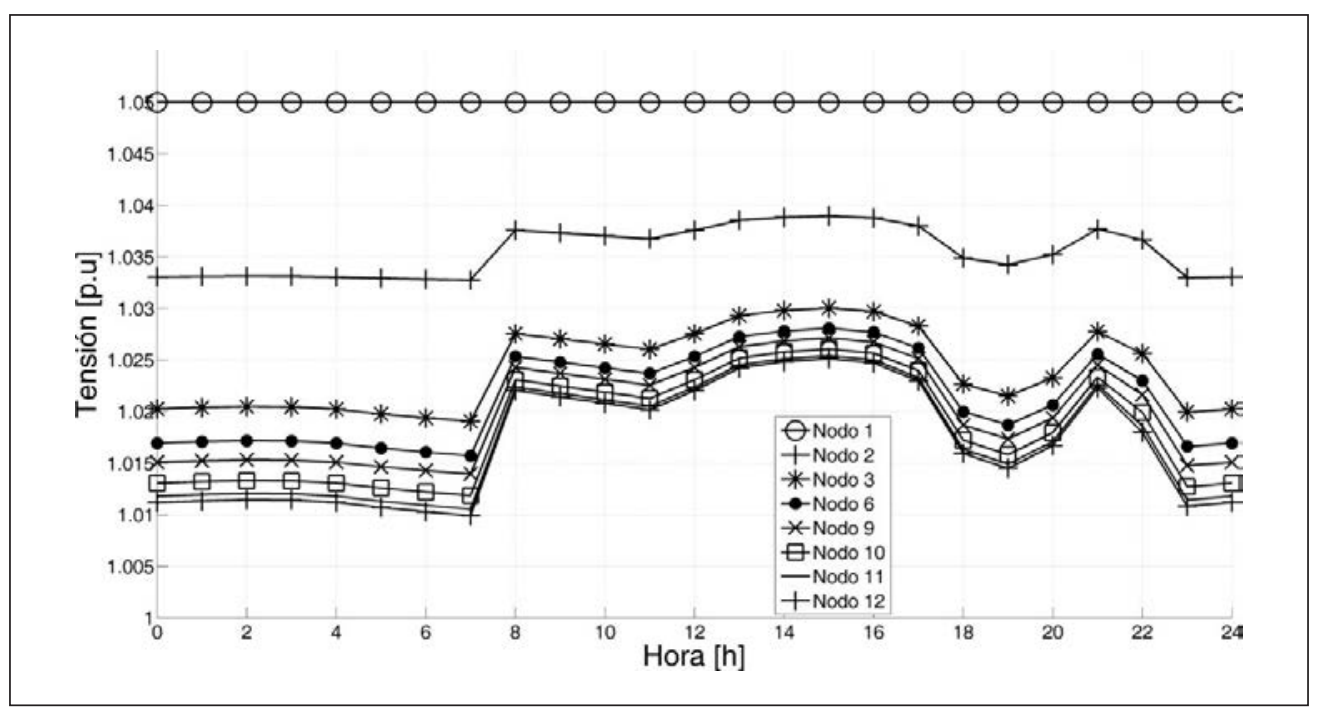

Figura 6. Perfiles de tensión incluyendo carga de VE

Fuente: elaboración propia. 


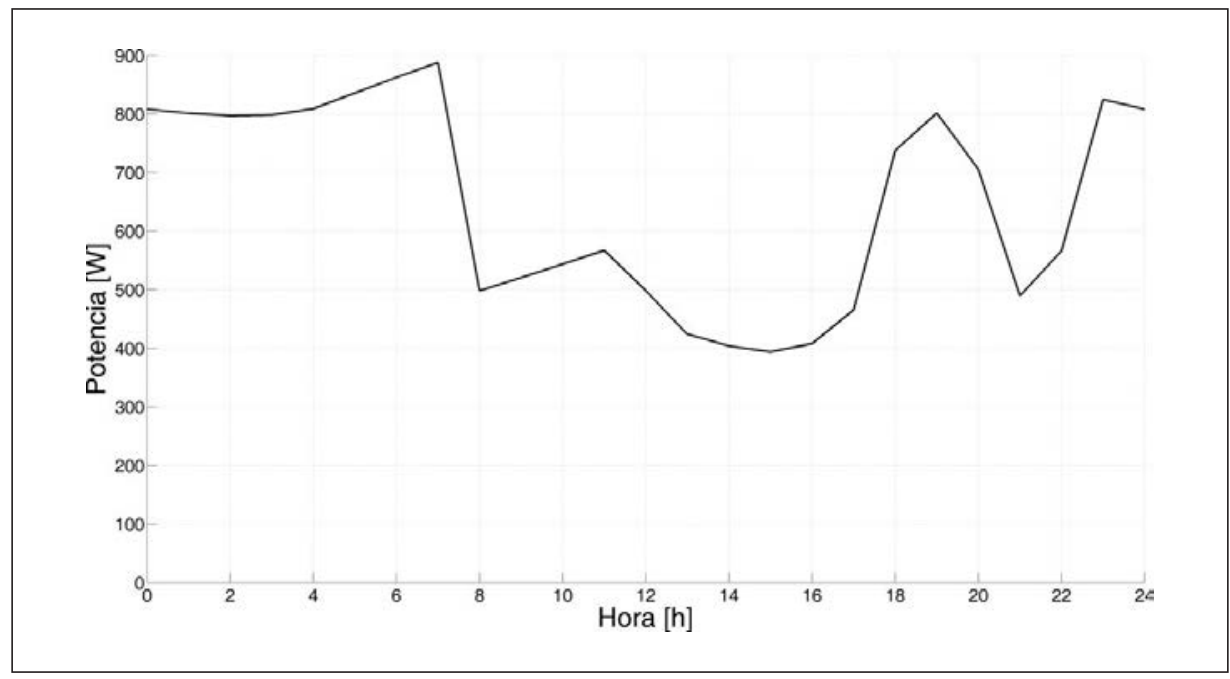

Figura 7. Pérdidas totales incluyendo carga de VE Fuente: elaboración propia.

Al igual que en el caso 1, la figura 8 presenta el comportamiento de los beneficios del comercializador, los ingresos por tarifa y los costos de la compra de energía en bolsa. En este caso se evidencia un incremento en los beneficios obtenidos por el comercializador entre las horas 22-24 y 0-8. Los ingresos diarios corresponden a 287 MCOP, los costos de la compra de la energía consumida por la demanda 190 MCOP y el comercializador obtiene 97 MCOP diarios en beneficios.

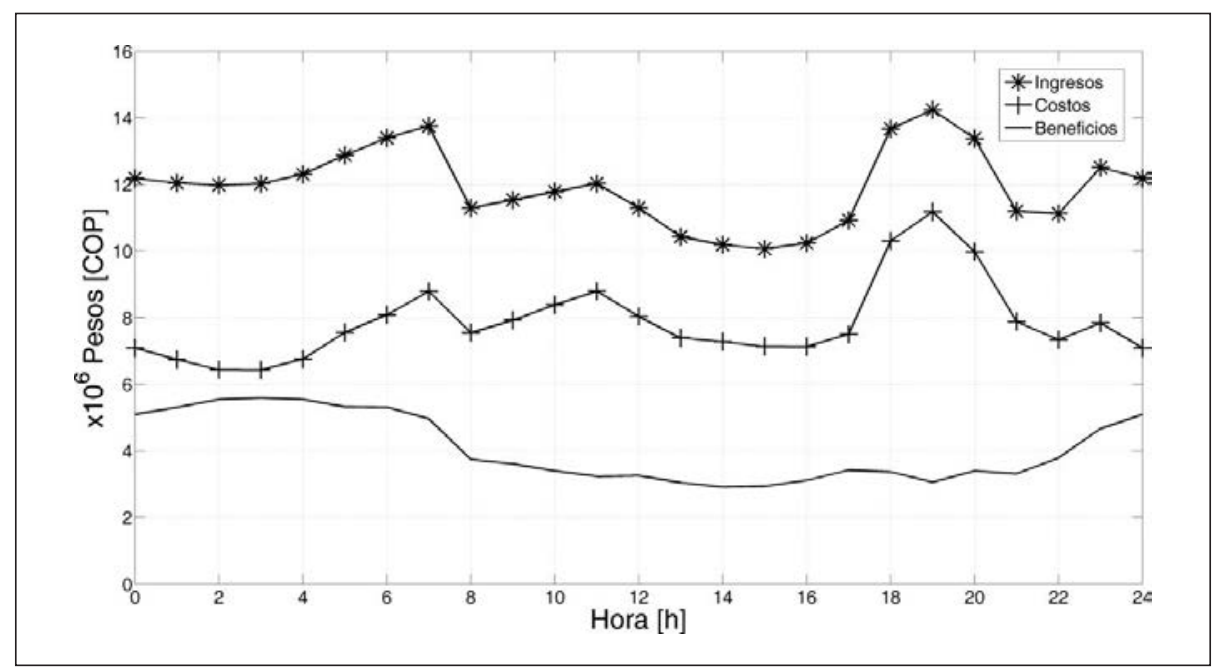

Figura 8. Beneficios del comercializador incluyendo carga de VE

Fuente: elaboración propia. 


\section{Caso 3: operación incluyendo carga y descarga de VE.}

En este caso se analizó el sistema de distribución permitiendo la carga y la descarga de los VE, esto es, se consideró que los VE contaban con tecnología V2G y se empleó el modelo 3 presentado en la sección 1. Los VE fueron incluidos en los mismos nodos que se emplearon para el caso 2. Las figuras 9 y 10 muestran las tensiones y las pérdidas en el ramal. Al comparar estas figuras con las obtenidas en el caso 1, es posible ubicar la carga y la descarga de VE por medio de sus impactos sobre la red. Las disminuciones en la tensión y el aumento de las pérdidas en horas de bajo consumo, indican los tiempos de carga de los VE, mientras que, el aumento de las tensiones y la disminución de las pérdidas señalan los tiempos de descarga. En este caso las pérdidas diarias de energía ascienden a los 16,1 kWh. Al comparar el resultado anterior con el obtenido en el caso 2, se aprecia que la descarga de la energía almacenada por los VE en la red no necesariamente aumenta la eficiencia del sistema. Esto se debe a que el precio de bolsa tiene más peso sobre los tiempos de carga y descarga de los VE que las pérdidas en la maximización del beneficio, es decir, un comercializador programaría la recarga de los VE en horas en las que el precio de bolsa es bajo, aunque las pérdidas del sistema de distribución sean altas.

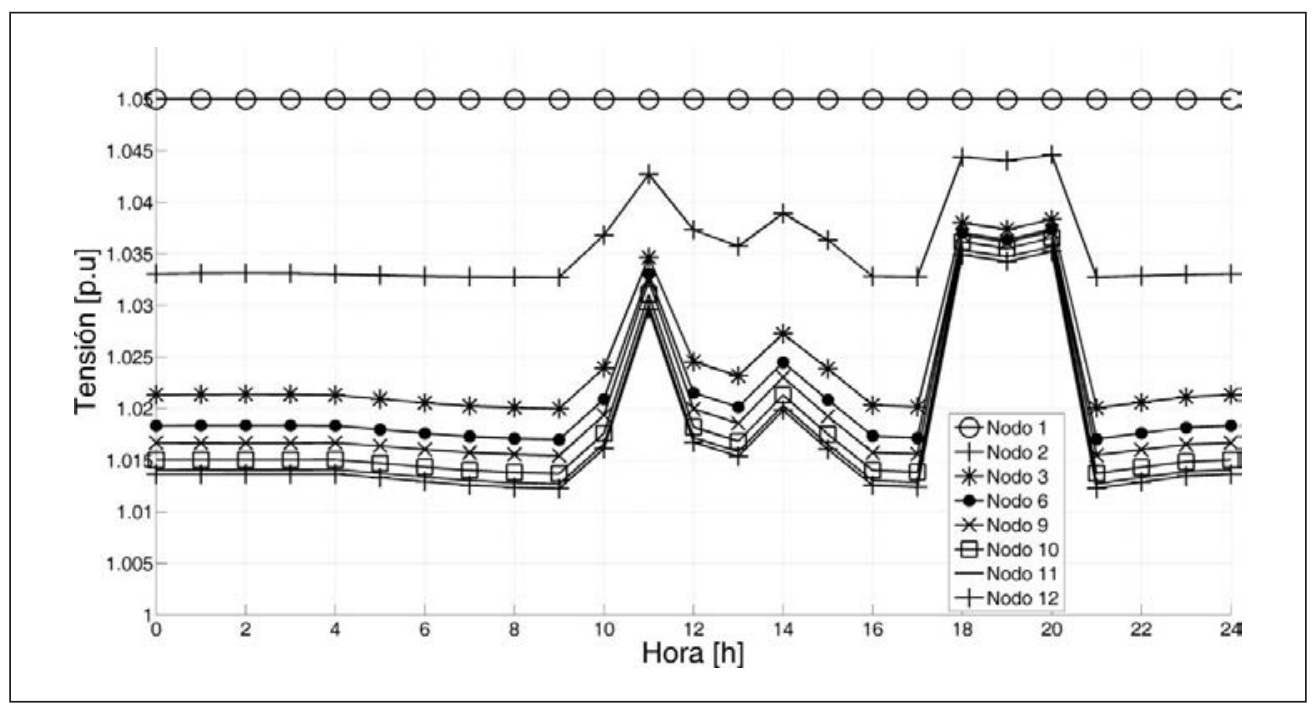

Figura 9. Perfiles de tensión incluyendo carga y descarga de VE Fuente: elaboración propia.

Por otro lado, la figura 11 muestra una disminución del beneficio del comercializador en las horas de alto consumo debido a la descarga de VE. En este caso, se nota una disminución en la hora 11 y entre las horas 17-19 coincidiendo con la descarga de los VE. Esto también coincide con una mejora en los perfiles de tensión y menores 
pérdidas. Finalmente, se presenta un incremento en los beneficios obtenidos por el comercializador entre las horas 22-24 y 0-8. Los ingresos diarios corresponden a 286,6 MCOP, los costos de la compra de la energía consumida por la demanda 188,5 MCOP y el comercializador obtiene 98,1 MCOP diarios en beneficios.

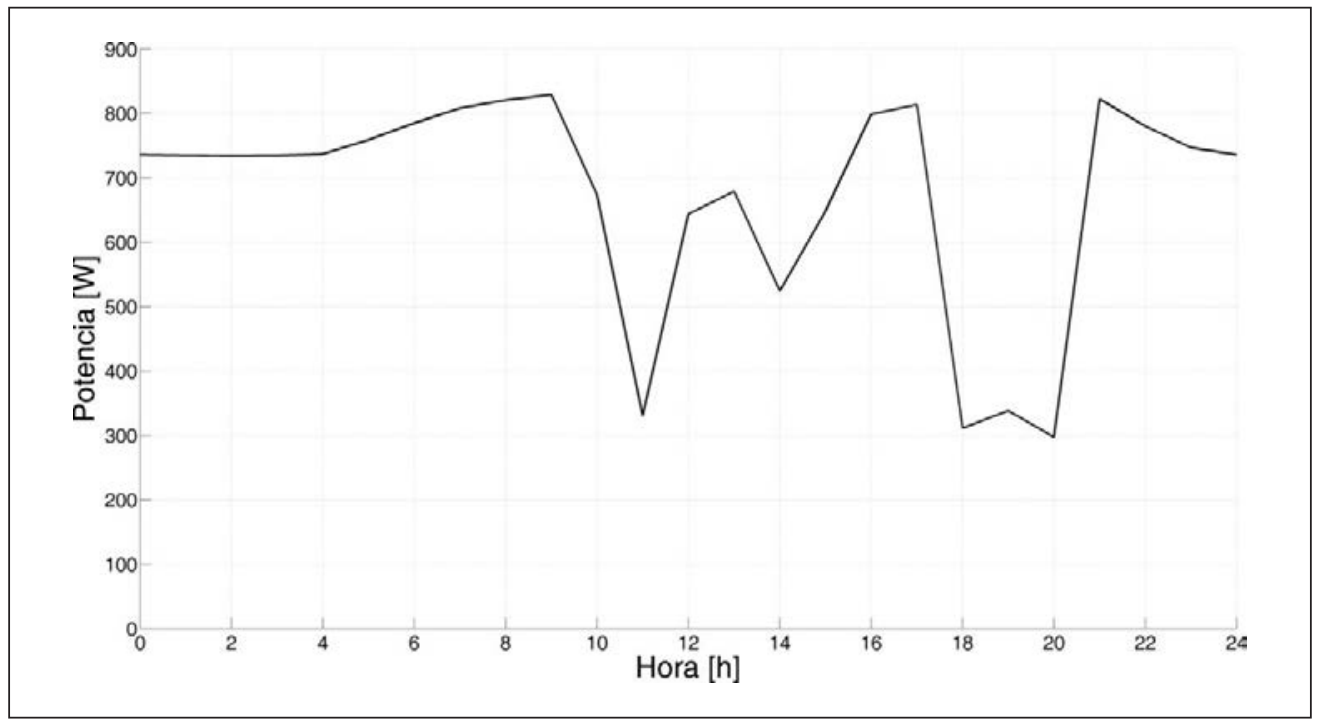

Figura 10. Pérdidas totales incluyendo carga y descarga de VE Fuente: elaboración propia.

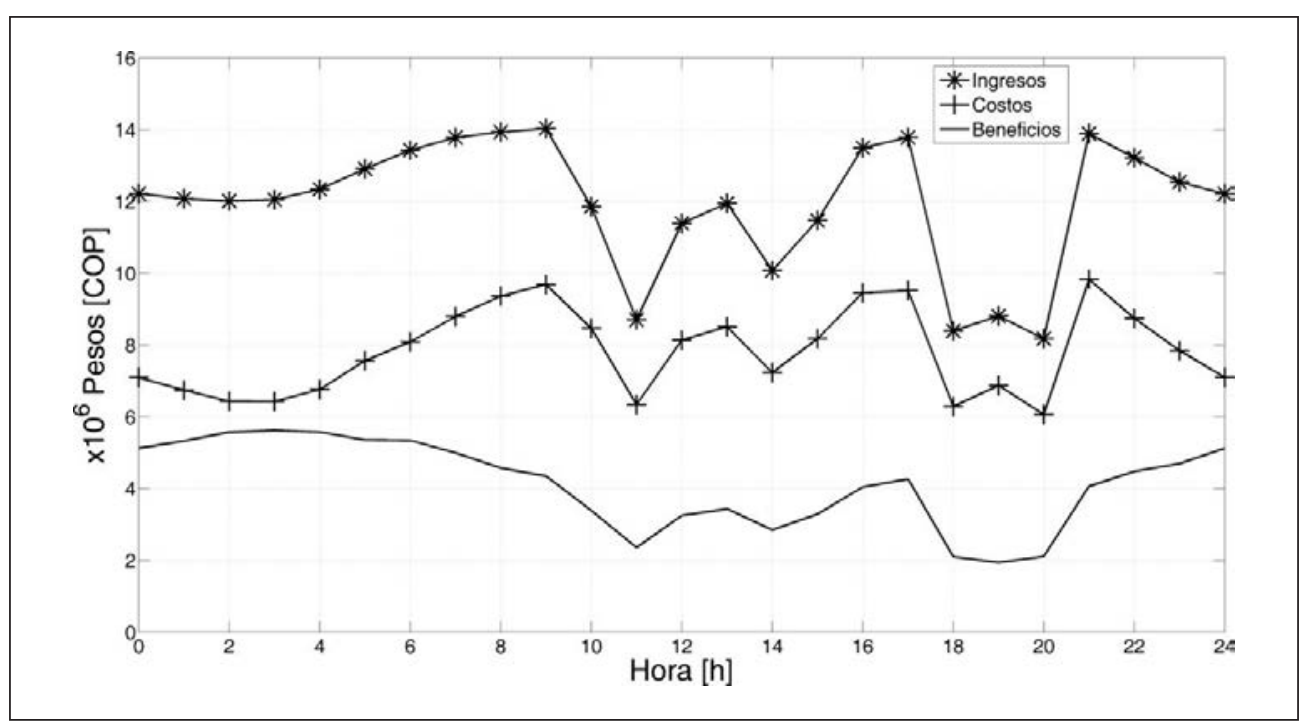

Figura 11. Beneficios del comercializador incluyendo carga y descarga de VE Fuente: elaboración propia. 


\section{DISCUSIÓN}

En términos técnicos, los VE representarían un incremento en las pérdidas del sistema y el consumo de energía, deteriorando los perfiles de tensión en los momentos de carga pero con la posibilidad de mejorarlos durante la descarga. Por otro lado, en términos económicos, los comercializadores podrían beneficiarse de una masificación de los VE en Colombia al incrementar la venta de energía a los usuarios, principalmente en horas que típicamente han sido de bajo consumo.

Aunque en este trabajo la inclusión de VE con tecnología V2G presenta un mayor beneficio económico para los comercializadores, este aumento solo representa un incremento del 1,1 \% cuando se compara con VE que solo cuentan con posibilidades de recarga. Este pequeño incremento en el beneficio podría lograrse a expensas de una fuerte inversión en infraestructura y un aumento de la complejidad en la operación del sistema, por lo que es necesario realizar un análisis económico más detallado para definir la viabilidad de la tecnología V2G desde el punto de vista de un comercializador. De cualquier forma, los resultados indican que los comercializadores podrían contar con incentivos adicionales para el fomento de los VE en Colombia.

Adicional a los resultados presentados, se obtuvo que el circuito simulado opera dentro de los límites técnicos cuando se tiene una inclusión de VE del 25 \%. Para un número mayor de VE la corriente en el alimentador excede los límites operativos. En este sentido, resulta necesario planear de manera conjunta la masificación de VE, la ampliación de la infraestructura de redes secundarias, la construcción de circuitos dedicados exclusivamente a la carga de VE, estrategias que procuren la recarga inteligente por parte de los usuarios, y la posibilidad de la coordinación de la carga y descarga de los VE desde los OR.

Por último, los resultados obtenidos en este trabajo presentan un escenario positivo para los comercializadores dado que los modelos propuestos procuran la maximización de su beneficio. Es decir, los modelos empleados para la carga y descarga de los VE no han considerado los hábitos de conexión y desconexión que podrían presentar los usuarios en Colombia. Puntualmente, las estrategias de carga y descarga que podría emplear un comercializador para maximizar su beneficio podrían cambiar según el día de la semana y dependiendo de si el circuito es rural o urbano. La inclusión de los hábitos y el tipo de circuito se propone para ser desarrollado en trabajos futuros.

\section{CONCLUSIONES}

La necesidad de diversificar la canasta energética colombiana ha permitido generar iniciativas que apuntan hacia una mayor participación de los VE en el mercado de los vehículos automotores. Este aumento podría generar impactos técnicos y económicos en 
las redes de distribución de energía eléctrica que afectarían a comercializadores y OR. Los resultados que se han presentado en este trabajo indican que los impactos técnicos y económicos dependen de la tecnología con que cuenten los VE que se conectan a la red, esto es, dependen de si los VE cuentan o no con tecnología V2G.

Contrario a lo que podría pensarse de manera intuitiva, los resultados muestran que la implementación de tecnología V2G no necesariamente aumenta la eficiencia de la red. De hecho, el criterio de decisión empleado por los comercializadores para la coordinación entre la carga y descarga de los VE podría estar influenciado más por el incremento del consumo de energía y las variaciones del precio de bolsa, que por los cambios en las pérdidas del sistema. En este sentido, la maximización del beneficio del comercializador obedecería más a criterios económicos que a criterios técnicos cuando se ignoran los cambios en el corto plazo del costo unitario de prestación del servicio. De cualquier forma, los comercializadores y los OR obtendrían beneficios adicionales del uso de VE en Colombia, que deberían ser tenidos en cuenta al momento de fijar políticas y aspectos regulatorios orientados a la masificación de VE en el país.

\section{REFERENCIAS}

[1] International Energy Agency, “Global EV Outlook 2016. Beyond one million electric cars,” [En línea], Disponible http://selectra.ie/sites/default/files/pdf/

Global_EV_Outlook_2016.pdf

[2] Hanke C., Hüelsmann M., Fornahl D. "Socio-Economic Aspects of Electric Vehicles: A Literature Review”, en Hülsmann M., Fornahl D. Evolutionary Paths Towards the Mobility Patterns of the Future. Lecture Notes in Mobility. Springer, Berlin, Heidelberg, pp 13 - 36, 2014

[3] COP21, "Paris Declaration on Electro-Mobility and Climate Change and Call to Action," presentado en Un Climate Change Conference, París, 2015.

[4] B. Morales, Modelo de masificación de vehículos eléctricos en Bogotá D.C., Bogotá D.C.: Universidad Nacional de Colombia, 2014.

[5] Ministerio de Comercio, Industria y Turismo, Decreto número 2909 de 17 de diciembre de 2013, Bogotá D.C., 2013.

[6] Unidad de Planeación Minero Energética (UPME), "Plan Energético Nacional Colombia: Ideario Energético 2050,” Bogotá, 2015.

[7] L. Pieltain et al., "Assessment of the Impact of Plug-in Electric Vehicles on Distribution Networks,” IEEE Transactions on power systems, vol. 26, n. ํ 1, pp. 206-213, 2016.

[8] K. Clement-Nyns et al., "The Impact of Charging Plug-In Hybrid Electric Vehicles on a Residential Distribution Grid,” IEEE Transactions on power systems, vol. 25, n. ${ }^{\circ}$ 1, pp. 371-380, 2010. 
[9] G. Putrus et al., "Impact of Electric Vehicles on Power Distribution Networks," presentado en IEEE Vehicle Power and Propulsion Conference, 2009, Dearborn, 2009.

[10] A. Arias et al., "Optimal Charging Schedule of Electric Vehicles Considering Variation of Energy Price," presentado en Transmission and IEEE Distribution Conference and Exposition - Latin America (PES TandD-LA), 2014 IEEE PES, Medellín, 2014.

[11] Banco Interamericano de Desarrollo, “La incorporación de los vehículos eléctricos en América Latina,” BID, 2016.

[12] Comisión de Regulación de Energía y Gas (CREG), Resolución CREG 042 de 1999, Bogotá, 1999.

[13] Comisión de Regulación de Energía y Gas (CREG), Resolución CREG 097 de 2008, Bogotá, 2008.

[14] A. Rueda-Medina et al., "A mixed-integer linear programming approach for optimal type, size and allocation of distributed generation in radial distribution systems," Electric Power System Research, vol. 97, pp. 133-143, 2013.

[15] J. Franco et al., "Optimal allocation of capacitors in radial distribution systems with distributed generation,” presentado en 2011 IEEE PES Conference on Innovative Smart Grid Technologies (ISGT Latin America), Medellín, 2011.

[16] G. Marulanda et al., "Análisis cualitativo del impacto de la respuesta de la demanda en los cargos por uso del sistema de distribución,” Ingeniería y competitividad, vol. 19, n. 1, pp. 147-158, 2017.

[17] C. Peng et al., "An optimal dispatching strategy for V2G aggregator participating in supplementary frequency regulation considering EV driving demand and aggregator's benefits," Applied Energy, vol. 190, pp. 591-599, 2017.

[18] R. Rosenthal y GAMS Development Corporation, A User's Guide, Washington D.C.: GAMS Development Corporation, 2017.

[19] S. Ramírez, Redes de distribución de energía, Manizales, Manizales: Universidad Nacional de Colombia, 2004.

[20] XM S.A. E.S.P., “Informes de despacho”. [En línea], Disponible: https://www.xm.com.co/ Paginas/Generacion/informes-despacho.aspx

[21] K. Rojas y J. Nieto, Análisis de flujo de potencia y perfiles de tensión en sistemas de distribución ante distintos niveles de inclusión de vehículos eléctricos, Bogotá: Universidad de Lasalle, 2016. 